\title{
The Dilemma of Bilateral Tubal Pregnancy: Case Report and Literature Review
}

\section{Azzam HM*, Dayyana S and Tomlinson AJ}

Royal Bolton Hospital, Bolton, Lancashire, England

\section{Case Report}

This is a case of a 28 -year-old lady, nulliparous lady, with a two year history of primary infertility. Shehad undergone infertility investigations which revealed an ovulation with low day 21 progesterone assay. Pelvic ultrasound scanning showed a normal uterus and endometrium, bulky left ovary and normal right ovary. Laparoscopy, hysteroscopy and tubal dye test revealed mild to moderate endometriosis, probable chocolate cyst on the left ovary, tortuous and blocked left tube. However, there was a normal and patent right tube. Her husband's seminal analysis was sub-optimal with a good count of 47 million sperm $/ \mathrm{ml}$. However, only $7 \%$ normal forms and $4 \%$ were with rapid progressive motility. The diagnosis of sub fertility in both partners was made.

In order to treat the endometriosis the GnRH analogue leuprorelin acetate was administered for six months and the partner was referred to an urologist. Ten months later the patient after completing the course of GnRH analogue was reviewed. She had had three regular cycles, and the partner's seminal analysis had shown improvement. However, day 21 progesterone level was still showing anovulation.

Therefore, ovulation induction was commenced using $50 \mathrm{mg}$ clomiphene citrate cycle day 2-6, ovulation monitored by ultrasonic folliculometry and day 21 progesterone level. Ovulation was achieved at $100 \mathrm{mg}$ clomiphene citrate. However, the patient failed to conceive with 6 months of therapy. At this stage the couples were referred for In Vitro Fertilization (IVF)

Several months later the couple started an IVF treatment in a private clinic. Fifty-seven eggs were retrieved in the first cycle of which 16 were fertilized by Intra-cytoplasmic Sperm Injection (ICSI). The first cycle was not successful. However, further treatment was given using embryos that had been cryopreserved, two embryos being transferred.

Eighteen days after the embryo transfer the patient was admitted to the hospital as an emergency with history of lower abdominal pain of one-day duration. Physical examination was satisfactory with only mild tenderness in the right iliac fossa.

On admission a pregnancy test was positive. Ultrasound scan performed the next day showed a uterus with no evidence of gestation, a rather bulky right ovary but with no significant cysts, there was not any pelvic free fluid or other adrenal mass.

The patient remained an inpatient under observation. Serum BhCG was reported the next day to be $1648 \mathrm{iu} / \mathrm{l}$ and $1840 \mathrm{iu} / \mathrm{l} 48$ hours later. In view of persistent symptoms and unsatisfactory rise in the BhCG, the patient underwent diagnostic laparoscopy on day 5 of here admission. She was found to have a right tubal pregnancy and left tubal

hydrosalpinx. Therefore, bilateral salpingectomy was performed. The histopathology of both resected tubes confirmed the existence of bilateral ectopic gestation. The patient was kept in hospital for 2 more days where she made a satisfactory postoperative recovery. The patient became pregnant with twins following further IVF treatment and went on to have normal vaginal delivery of these babies, who are fit and well.

\section{Discussion}

Ectopic pregnancy is still a leading cause of pregnancy related death during first trimester with a rate of 1.8 per 1000 ectopic pregnancies [1]. The incidence of ectopic pregnancy is around 4.5-16.8 per 1000 pregnancies, the incidence has nearly tripled in the last few years largely due to an increased incidence of sexually transmitted diseases and assisted reproductive techniques (IVF) [2]. Ectopic pregnancy is a recognised risk of IVF treatment with the incidence being 45 in 1000 clinical pregnancies after IVF [3].

Bledsoe reported the first case of bilateral tubal pregnancy in 1918 [2]. Bilateral tubal pregnancy is still a rare phenomenon varying in frequency between 1 per 725 and 1 per 1580 ectopic pregnancies [4] and this incident increases with IVF treatment to be around 2 per 1000 clinical pregnancies (45 per 1000 ectopic pregnancies) [3].

Diagnosis of bilateral tubal pregnancy is more complicated and difficult than unilateral ectopic pregnancy although following the same principles and continues to be an important challenge facing gynaecologists, as when one ectopic is found that could be assumed to be the cause for all the patent's symptoms.

In our unit a clinical algorithm is used in the management of bleeding and pain in early pregnancy, and the patient was managed accordingly. Due to suboptimal rise in BhCG, no intrauterine pregnancy by ultrasound scan and persistent symptoms laparoscopy was performed.

During our review of the literature no figures could be found describing BhCG levels in bilateral ectopic pregnancy. However, it is well documented that BhCG levels following IVF treatment almost double in intra uterine twin pregnancy when compared with singleton pregnancy. Therefore, one would expect similar increase in bilateral tubal pregnancy compared to one tubal pregnancy. Similarly with respect to transvaginal ultrasound, diagnosis of a singleton pregnancy can be visualised with a lower level of BhCG than in a multiple pregnancy.

This case demonstrates not only the prevalence and difficulty in diagnosing bilateral tubal pregnancy but also demonstrates the limitations of ultrasound and laparoscopy in making such a diagnosis. Since the left tube in this case was only removed because of the abnormality noted not because an ectopic pregnancy was suspected.

*Corresponding author: Azzam HM, Royal Bolton Hospital, Bolton, Lancashire England, E-mail: HAzzam@BRHA.mb.ca

Received August 17, 2012; Accepted September 11, 2012; Published September 13, 2012

Citation: Azzam HM, Dayyana S, Tomlinson AJ (2012) The Dilemma of Bilatera Tubal Pregnancy: Case Report and Literature Review. J Clin Case Rep 2:198. doi:10.4172/2165-7920.1000198

Copyright: (c) 2012 Azzam HM, et al. This is an open-access article distributed under the terms of the Creative Commons Attribution License, which permits unrestricted use, distribution, and reproduction in any medium, provided the original author and source are credited. 
In view of the above, would a prophylactic laparoscopic bilateral salpingectomy for all women with or even without tubal disease prior to In Vitro Fertilization (IVF) be a solution to this dilemma of bilateral tubal pregnancy. There is evidence in the literature supporting this approach to improve pregnancy outcome especially in the presence of a damaged tube which would have a negative effect on In Vitro Fertilization (IVF) outcome [5]. However, this approach failed to reduce ectopic pregnancy rate [6] and bilateral salpingectomy may have an adverse effect on ovarian response to ovulation induction in an IVF treatment [7]. Moreover, these patients are at particular risk for corneal pregnancy instead [8] and this approach would also result in unnecessary surgery.

Therefore, clinician's intuition, high index of suspension and a very close inspection of the abdomen, pelvis and contra lateral tube during a surgery (laparoscopy or laparotomy) for ectopic pregnancy remain crucial in the diagnosis of bilateral tubal pregnancy, if any abnormality of the contra lateral tube is present there could be serious consideration for removal that tube also.

\section{References}

1. (1999) The management of tubal pregnancy. RCOG, Guidelines (21).
2. Bustos Lopez HH, Rojas-Poceros G, Barron Vallejo J, Cintora Zamudio S Kably Ambe A, et al. (1998) [Conservative laparoscopic treatment of bilateral ectopic pregnancy. 2 case reports and review of the literature]. Ginecol Obste Mex 66: 13-17.

3. Marcus SF, Brinsden PR (1995) Analysis of the incidence and risk factors associated with ectopic pregnancy following in-vitro fertilization and embryo transfer. Hum Reprod 10: 199-203.

4. Rondeau JA, Hibbert ML, Nelson KM (1997) Combined tubal and cornual pregnancy in a patient without risk factors. A case report. J Reprod Med 42: 675-677.

5. Chang CC, Wu TH, Tsai HD, Lo HY (1998) Bilateral simultaneous tuba sextuplets: pregnancy after in-vitro fertilization--embryo transfer following salpingectomy. Hum Reprod 13: 762-765.

6. Johnson NP, Mak W, Sowter MC (2002) Laparoscopic salpingectomy for women with hydrosalpinges enhances the success of IVF: a Cochrane review. Hum Reprod 17: 543-548.

7. Lass A, Ellenbogen A, Croucher C, Trew G, Margara R, et al. (1998) Effect of salpingectomy on ovarian response to superovulation in an in vitro fertilizationembryo transfer program. Fertil Steril 70: 1035-1038.

8. Agarwal SK, Wisot AL, Garzo G, Meldrum DR (1996) Cornual pregnancies in patients with prior salpingectomy undergoing In Vitro Fertilization and embryo transfer. Fertil Steril 65: 659-660. 